\title{
Location Assisted Subcarrier and Power Allocation in Underlay Mobile Cognitive Radio Networks
}

\author{
Muhammad Farooq-I-Azam ${ }^{1}$, Wenjuan $\mathrm{Yu}^{2}$, Qiang $\mathrm{Ni}^{3}$, Mianxiong Dong ${ }^{4}$ and Atta ul Quddus ${ }^{2}$ \\ ${ }^{1}$ Department of Electrical Engineering, COMSATS University Islamabad, Lahore Campus, Pakistan. \\ ${ }^{2}$ Department of Electrical and Electronic Engineering, University of Surrey, UK. \\ ${ }^{3}$ InfoLab21, School of Computing \& Communications, Lancaster University, UK. \\ ${ }^{4}$ Department of Information and Electronic Engineering, Muroran Institute of Technology, Japan.
}

\begin{abstract}
In this paper we perform subcarrier and power allocation for the downlink transmission in a cognitive radio network by exploiting the location information of mobile secondary users. A mixed integer nonlinear problem is formulated which maximizes the aggregate capacity of the secondary network, subject to the constrained interference at the primary user and the maximum transmit power limit of the secondary base station. To solve the formulated problem, an optimal subcarrier allocation is first obtained based on a practical assumption, followed by an optimal power allocation achieved using standard optimization methods. The proposed solution can facilitate to reduce latency and provide high speed communication of big data for mobile secondary users since speed, direction and location are all taken into account for effective allocation of resources. Simulation results verify our design intentions and confirm the efficiency of the proposed resource allocation strategy.
\end{abstract}

\section{INTRODUCTION}

Tremendous increase in the usage of wireless communication and resulting wireless big data [1] has spurred usage of high number of frequency bands. Therefore, future generations of wireless networks may face radio spectrum scarcity issues due to the fixed allocation of licensed spectrum bands. The licensed spectrum remains vacant during the idle period of primary users (PUs) which results in its inefficient utilization. Therefore, technologies, such as cognitive radio (CR) [2] and device to device (D2D) communication [3], [4] are being developed to improve bandwidth and spectral efficiency. In particular, CR has been proposed as a promising solution which aims to opportunistically and efficiently utilize the spectrum by dynamically accessing frequency resources with the help of methods such as those provided by big spectrum data [1]. Orthogonal frequency division multiple access (OFDMA) in cognitive radio networks (CRN) allows the secondary users (SUs) to flexibly utilize the available licensed spectrum as long as their transmission does not interfere with that of the PUs. This can be managed by use of optimum transmit power allocation strategy so that the interference caused by SUs does not exceed the interference tolerance level of PUs. At the same time, it is desirable that the power allocation results in the maximization of throughput to ensure reliability and quality of service (QoS) to the SUs.

This research was supported in part by JSPS KAKENHI Grant JP16K00117, the KDDI Foundation, the Royal Society project IEC170324, EPSRC project EP/K011693/1, EPSRC IAA project CSA7113, and EU FP7 CROWN project PIRSES GA-2013-610524.
Opportunistic spectrum access protocol design and resource allocation algorithms have been extensively investigated for stationary SUs [2], [5]-[8]. For example, a distributed resource allocation algorithm using amplify and forward multi-antenna relays in a cooperative CRN is proposed in [5]. With the channel state information (CSI) available at the SU, an optimal resource allocation is obtained. In [2], a closed form analytical solution based upon Nash bargaining solution for the joint channel and power allocation problem in CRN is proposed. The proposed solution maximizes the overall throughput of the $\mathrm{CRN}$ while minimum rate requirements and efficient distribution of power among SUs are also guaranteed. A solution to the joint spectrum sensing and power allocation problem is proposed in [6]. In a similar work [7], the joint spectrum sensing and resource allocation problem for a downlink CRN is solved using monotonic optimization techniques.

In most of the previous work, such as [2], [5]-[8], mobility of SUs is not considered and the CSI is assumed to be constant. In reality, condition of the communication channel between the secondary base station (SBS) and the mobile SU changes at each instant of time. Hence, these resource allocation algorithms are applicable only to static environments. Similarly, location awareness is one of the six important cognitive abilities of a CRN identified by Federal Communications Commission (FCC) in [9] for efficient and flexible spectrum usage. However, it has not been extensively exploited in the existing literature to address resource allocation in CRNs.

In our work, we exploit the location cognitive ability of a CRN to predict the CSI of a mobile SU for efficient resource allocation. Specifically, we propose a novel method to predict the position of an SU in the next resource allocation time slot by using the position coordinates of the mobile SU at previous and current instants of time. We then utilize the predicted location information for the prediction of the CSI of the communication and interference links. Finally, the subcarrier and power allocation problem is solved using standard optimization techniques to maximize the average throughput by taking the shadow fading, predicted SU location, maximum power limit and interference constraint into consideration.

\section{SySTEM MODEL}

We consider downlink transmission in a CRN underlaid to a primary network (PRN). The CRN exploits OFDMA sub- 
channels for transmission such that the interference caused by the SU transmission remains below the permissible threshold of the PRN. An individual PU is represented by $p$ such that $p \in \mathbf{P}=\{1,2,3, \ldots, P\}$ where $\mathbf{P}$ is the set of PUs with total members $P$. Similarly, an arbitrary $\mathrm{SU}$ is represented by $q$ such that $q \in \mathbf{Q}=\{1,2,3, \ldots, Q\}$ where $\mathbf{Q}$ is the set of SUs with total members $Q$. The number of OFDMA subchannels available to the CRN is $N$ with a total bandwidth of $B H_{z}$. Hence, the bandwidth of each subchannel is $B / N H_{z}$. An individual subchannel is represented by $n$ and the set of subchannels is given by $\mathbf{N}=\{1,2,3, \ldots, N\}$ with total members $N$. To limit interference and to ensure reliable data transmission, each subchannel $n$ is assigned exclusively to a single SU $q$. Secondary subchannel allocation index is denoted by $z_{q n}$ which is a member of the vector $\mathbf{z}=\left[z_{11}, z_{12}, z_{13}, \ldots, z_{q n}\right]$. It has a binary value and $z_{q n}=1$ if the channel $n$ is allocated to an $\mathrm{SU} q$ and $z_{q n}=0$ otherwise. The power allocated to an SU $q$ on subchannel $n$ is denoted by $P_{q n}^{S}$ while the maximum transmission power of the SBS is $P_{\max }$. The threshold of interference permissible at PU $p$ is represented by $I_{p t}$. The corresponding threshold value of signal to interference noise ratio (SINR) at a PU is $\beta$. For the interference to remain below threshold $I_{p t}$, the SINR $\gamma_{p}$ of the signal transmitted by the primary base station (PBS) received at the PU $p$ should be such that $\gamma_{p} \geq \beta$.

We consider an urban environment with mobile SUs. We model large scale fading by using log-normal distribution for the estimation of path loss and shadowing. The small scale fading and multipath arising from network mobility are modeled using Rayleigh fading. The CRN has knowledge of location information using localization algorithms, such as, [10]. We assume that a mobile SU maintains its speed and direction during resource allocation period.

\section{A. SINR at PU}

The interference $I_{p n}^{S}$ caused by the communication between the SBS and an SU $q$ operating at subchannel $n$ to a PU $p$ is

$$
I_{p n}^{S}=h_{p n}^{S} P_{q n}^{S},
$$

where $h_{p n}^{S}$ is the gain of the interference link between the SBS and the PU $p$. The interference $I_{p n}^{S}$ can also be calculated using the power spectral density (PSD) of the OFDM subcarrier [11]. Using $I_{p n}^{S}$ from (1), the SINR $\gamma_{p}$ at PU $p$ is given by

$$
\gamma_{p}=\frac{h_{p n}^{P} P_{n}^{P}}{I_{p n}^{S}+\frac{B}{N} N_{o}},
$$

where $h_{p n}^{P}$ is the gain of the PRN communication link between the PBS and the PU $p$ on subchannel $n, P_{n}^{P}$ denotes the PBS power transmitted on subchannel $n$ and $N_{o}$ is the noise power spectral density. From (2), the threshold value $I_{p t}$ of interference $I_{p n}^{S}$ permissible at a PU $p$ such that SINR $\gamma_{p} \geq \beta$ is given by

$$
I_{p t}=\frac{1}{\beta}\left(h_{p n}^{P} P_{n}^{P}\right)-\frac{B}{N} N_{o}
$$

\section{B. SINR at SU}

We consider a mobile $\mathrm{SU} q$ traveling with an average speed $v_{q}$ as illustrated in Fig. 1. It starts from a point $\left(x_{a}, y_{a}\right)$ at time $t_{a}$ and arrives at $\left(x_{b}, y_{b}\right)$ at time $t_{b}$ and at $\left(x_{c}, y_{c}\right)$ at $t_{c}$. The distance elements between the SBS and the SU at the three positions are represented by $d_{s a}, d_{s b}$ and $d_{s c}$ respectively. Likewise, the distance elements between the PBS and the SU are denoted by $d_{p a}, d_{p b}$ and $d_{p c}$. We represent the gain of the wireless communication channel between the SBS and the SU $q$ at instants $t_{a}, t_{b}$ and $t_{c}$ at positions $\left(x_{a}, y_{a}\right)$, $\left(x_{b}, y_{b}\right)$ and $\left(x_{c}, y_{c}\right)$ by $h_{q n a}^{S}, h_{q n b}^{S}$ and $h_{q n c}^{S}$ respectively. The corresponding path loss is denoted by $P L_{s a}, P L_{s b}$ or $P L_{s c}$, and the gain due to the path loss is represented by $h_{s a p}, h_{s b p}$ or $h_{\text {scp }}$. Similarly, the gain of the interference link between the PBS and the mobile SU $q$ at instants $t_{a}, t_{b}$ and $t_{c}$ is denoted by $h_{q n a}^{P}, h_{q n b}^{P}$ and $h_{q n c}^{P}$ respectively. The path loss is denoted by $P L_{p a}, P L_{p b}$ or $P L_{p c}$, and the gain due to the path loss is represented by $h_{p a p}, h_{p b p}$ or $h_{p c p}$ depending upon the position of the SU $q$. Note that if the transmitted and received powers are denoted by $P_{t}$ and $P_{r}$ respectively, then the path loss is given by $P L=P_{t} / P_{r}$ and the gain due to the path loss is given by $P_{r} / P_{t}=1 / P L$. For the speeds under consideration, the time intervals between the instants $t_{a}, t_{b}$ and $t_{c}$ are short enough so that the multipath fading gain of a subchannel $n$, denoted by $h_{n}$, is assumed to be the same at $t_{a}, t_{b}$ and $t_{c}$. As the SU $q$ moves from $\left(x_{a}, y_{a}\right)$ to $\left(x_{b}, y_{b}\right)$, the instantaneous CSI of the communication and interference links can be obtained using the location information. Furthermore, the position $\left(x_{c}, y_{c}\right)$ of the $\mathrm{SU} q$ at $t_{c}$ is predicted considering its speed and direction as it travels from $\left(x_{a}, y_{a}\right)$ to $\left(x_{b}, y_{b}\right)$. This information is then used for the prediction of the CSI of communication and interference links to $\mathrm{SU} q$ at $t_{c}$.

We use log-normal shadowing model for estimation of the

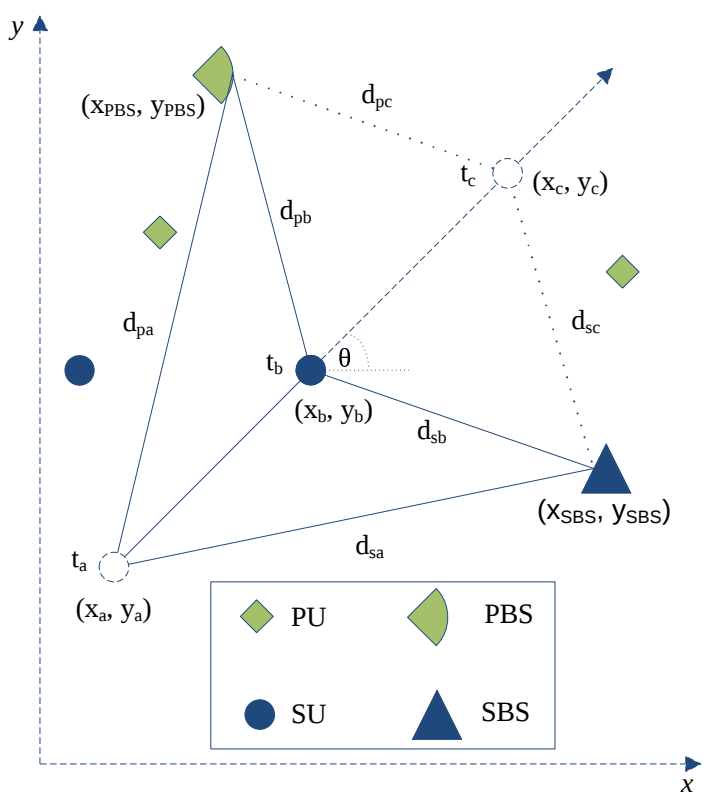

Fig. 1. System model of a CRN with mobile SUs. 
path loss. For example, the path loss $P L_{s a}$ between the SBS and the $\mathrm{SU} q$ at $\left(x_{a}, y_{a}\right)$ is expressed as

$$
P L_{s a}[d B]=\overline{P L}_{s}\left(d_{o}\right)+10 \alpha \log \left(\frac{d_{s a}}{d_{o}}\right)+\chi_{\sigma s a},
$$

where $\overline{P L}_{s}\left(d_{o}\right)$ is the average path loss at a reference distance $d_{o}$ from the SBS, $\alpha$ is the path loss exponent and $\chi_{\sigma s a}$ accounts for the shadow fading between the SBS and the SU. If the reference distance $d_{o}$ is the unit distance, in which case we denote $\overline{P L}_{s}\left(d_{o}\right)$ by $\overline{P L}_{s o}$, then

$$
P L_{s a}[d B]=\overline{P L}_{s o}+10 \alpha \log \left(d_{s a}\right)+\chi_{\sigma s a} .
$$

Let us now consider that the mobile SU $q$ whose initial position was $\left(x_{a}, y_{a}\right)$ at time $t_{a}$ is now at $\left(x_{b}, y_{b}\right)$ at time $t_{b}$. Using this information, the CRN can predict the instantaneous position $(x, y)$ of the $\mathrm{SU} q$ at a future time $t$ as below:

$$
\begin{aligned}
x(t)=x_{b} & +\frac{t-t_{b}}{t_{b}-t_{a}} \sqrt{\left(x_{b}-x_{a}\right)^{2}+\left(y_{b}-y_{a}\right)^{2}} \\
& \times \cos \left(\tan ^{-1} \frac{y_{b}-y_{a}}{x_{b}-x_{a}}\right), \\
y(t)=y_{b} & +\frac{t-t_{b}}{t_{b}-t_{a}} \sqrt{\left(x_{b}-x_{a}\right)^{2}+\left(y_{b}-y_{a}\right)^{2}} \\
& \times \sin \left(\tan ^{-1} \frac{y_{b}-y_{a}}{x_{b}-x_{a}}\right) .
\end{aligned}
$$

Using the predicted instantaneous position $(x, y)$, the instantaneous distance $d_{s}(t)$ between the SBS located at $\left(x_{S B S}, y_{S B S}\right)$ and the $\mathrm{SU} q$ is given by

$$
\begin{aligned}
d_{s}(t) & =\left(\left[x_{b}-x_{S B S}+\frac{t-t_{b}}{t_{b}-t_{a}} \sqrt{\left(x_{b}-x_{a}\right)^{2}+\left(y_{b}-y_{a}\right)^{2}}\right.\right. \\
& \left.\times \cos \left(\tan ^{-1} \frac{y_{b}-y_{a}}{x_{b}-x_{a}}\right)\right]^{2} \\
& +\left[y_{b}-y_{S B S}+\frac{t-t_{b}}{t_{b}-t_{a}} \sqrt{\left(x_{b}-x_{a}\right)^{2}+\left(y_{b}-y_{a}\right)^{2}}\right. \\
& \left.\left.\times \sin \left(\tan ^{-1} \frac{y_{b}-y_{a}}{x_{b}-x_{a}}\right)\right]^{2}\right)^{\frac{1}{2}} .
\end{aligned}
$$

In a similar manner, the instantaneous distance $d_{p}(t)$ between the PBS located at $\left(x_{P B S}, y_{P B S}\right)$ and the $\mathrm{SU} q$ is given by

$$
\begin{aligned}
d_{p}(t) & =\left(\left[x_{b}-x_{P B S}+\frac{t-t_{b}}{t_{b}-t_{a}} \sqrt{\left(x_{b}-x_{a}\right)^{2}+\left(y_{b}-y_{a}\right)^{2}}\right.\right. \\
& \left.\times \cos \left(\tan ^{-1} \frac{y_{b}-y_{a}}{x_{b}-x_{a}}\right)\right]^{2} \\
& +\left[y_{b}-y_{P B S}+\frac{t-t_{b}}{t_{b}-t_{a}} \sqrt{\left(x_{b}-x_{a}\right)^{2}+\left(y_{b}-y_{a}\right)^{2}}\right. \\
& \left.\times \sin \left(\tan ^{-1} \frac{y_{b}-y_{a}}{x_{b}-x_{a}}\right)^{2}\right)^{\frac{1}{2}} .
\end{aligned}
$$

The position $\left(x_{c}, y_{c}\right)$ of the SU $q$ at time $t_{c}$ can now be predicted by substituting $t=t_{c}$ in (6) and (7). Similarly, the distance $d_{s c}$ between the SBS and the SU $q$ and the distance $d_{p c}$ between the PBS and the SU $q$ at time $t_{c}$ can be estimated by substituting $t=t_{c}$ in (8) and (9) respectively. Using the predicted position and distance information, the path loss of the communication and interference links can be estimated similar to (4) and (5). Next, knowing the path loss, the instantaneous CSI of both the communication and interference links of the mobile SU can be calculated by the CRN. At instant $t_{b}$, the CSI in terms of the gain $h_{q n b}^{S}$ of the communication link and the interference power $I_{q n b}^{P}$ of the interfering link is given by

$$
\begin{gathered}
h_{q n b}^{S}=h_{n} h_{s b p}=\frac{h_{n}}{P L_{s b}}, \\
I_{q n b}^{P}=h_{q n b}^{P} P_{n}^{P},
\end{gathered}
$$

where

$$
h_{q n b}^{P}=h_{n} h_{p b p}=\frac{h_{n}}{P L_{p b}} .
$$

The SINR $\gamma_{q n b}$ at the SU q at $t_{b}$ is now given by

$$
\gamma_{q n b}=\frac{h_{q n b}^{S} P_{q n}^{S}}{I_{q n b}^{P}+\frac{B}{N} N_{o}} .
$$

It should be noted that $P L_{s b}$ and $P L_{p b}$ can be calculated similarly to (4) and (5). Likewise, the expected CSI at $t_{c}$ using the predicted position $\left(x_{c}, y_{c}\right)$ is given by

$$
\begin{gathered}
\mathbb{E}\left[h_{q n c}^{S}\right]=h_{n} h_{s c p}=\frac{h_{n}}{P L_{s c}}, \\
\mathbb{E}\left[I_{q n c}^{P}\right]=h_{q n c}^{P} P_{n}^{P},
\end{gathered}
$$

where

$$
h_{q n c}^{P}=h_{n} h_{p c p}=\frac{h_{n}}{P L_{p c}} .
$$

The expected value of SINR $\gamma_{q n c}$ at $t_{c}$ is then given by

$$
\mathbb{E}\left[\gamma_{q n c}\right]=\frac{\mathbb{E}\left[h_{q n c}^{S}\right] P_{q n}^{S}}{\mathbb{E}\left[I_{q n c}^{P}\right]+\frac{B}{N} N_{o}} .
$$

Dividing (14) by (10), we can express the expected CSI at predicted position $\left(x_{c}, y_{c}\right)$ in terms of known CSI at $t_{b}$.

$$
\begin{gathered}
\frac{\mathbb{E}\left[h_{q n c}^{S}\right]}{h_{q n b}^{S}}=\frac{P L_{s b}}{P L_{s c}} \\
10 \log \left(\frac{\mathbb{E}\left[h_{q n c}^{S}\right]}{h_{q n b}^{S}}\right)=P L_{s b}[d B]-P L_{s c}[d B] .
\end{gathered}
$$

However,

$$
\begin{aligned}
& P L_{s b}[d B]=\overline{P L}_{s o}+10 \alpha \log \left(d_{s b}\right)+\chi_{\sigma s b}, \\
& P L_{s c}[d B]=\overline{P L}_{s o}+10 \alpha \log \left(d_{s c}\right)+\chi_{\sigma s c} .
\end{aligned}
$$

Using these values in (19),

$$
\begin{aligned}
10 \log \left(\frac{\mathbb{E}\left[h_{q n c}^{S}\right]}{h_{q n b}^{S}}\right) & =10 \alpha \log \left(d_{s b}\right)-10 \alpha \log \left(d_{s c}\right) \\
& +\chi_{\sigma s b}-\chi_{\sigma s c} .
\end{aligned}
$$


Shadow fading $\chi_{\sigma s b}$ at $\left(x_{b}, y_{b}\right)$ and $\chi_{\sigma s c}$ at $\left(x_{c}, y_{c}\right)$ follow a normal distribution with a zero mean value so that

$$
\mathbb{E}\left[h_{q n c}^{S}\right]=\left(\frac{d_{s b}}{d_{s c}}\right)^{\alpha} h_{q n b}^{S} .
$$

In a similar manner, the expected value of interference at predicted position $\left(x_{c}, y_{c}\right)$ at $t_{c}$ can be derived in terms of known value of interference $I_{q n b}^{P}$ at time $t_{b}$ at position $\left(x_{b}, y_{b}\right)$ and the distances $d_{p b}$ and $d_{p c}$.

$$
\mathbb{E}\left[I_{q n c}^{P}\right]=\left(\frac{d_{p b}}{d_{p c}}\right)^{\alpha} I_{q n b}^{P} .
$$

Knowing the values of $h_{q n c}^{S}$ and $I_{q n c}^{P}$, the capacity of the subchannel $n$ between the SBS and the SU $q$ at $t_{c}$ at position $\left(x_{c}, y_{c}\right)$ is given by

$$
\begin{aligned}
\mathbb{E}\left[C_{q n c}\right] & =\frac{B}{N} \log _{2}\left(1+\mathbb{E}\left[\gamma_{q n c}\right]\right), \\
& =\frac{B}{N} \log _{2}\left(1+\frac{\mathbb{E}\left[h_{q n c}^{S}\right] P_{q n}^{S}}{\mathbb{E}\left[I_{q n c}^{P}\right]+\frac{B}{N} N_{o}}\right) .
\end{aligned}
$$

Using (23) and (24),

$$
\mathbb{E}\left[C_{q n c}\right]=\frac{B}{N} \log _{2}\left(1+\frac{\left(\frac{d_{s b}}{d_{s c}}\right)^{\alpha} h_{q n b}^{S} P_{q n}^{S}}{\left(\frac{d_{p b}}{d_{p c}}\right)^{\alpha} I_{q n b}^{P}+\frac{B}{N} N_{o}}\right) .
$$

\section{PROBlem Formulation}

By exploiting the location information of an $\mathrm{SU} q$ predicted using the speed and position information at the previous time slots, we aim to maximize the total average rate for the CRN, subject to the total transmission power limit and the maximum interference constraint at the PU.

$$
\max _{P_{q n}^{S}, z_{q n}} \frac{B}{N} \sum_{q=1}^{Q} \sum_{n=1}^{N} z_{q n} \mathbb{E}\left[\log _{2}\left(1+\frac{\left(\frac{d_{s b}}{d_{s c}}\right)^{\alpha} h_{q n b}^{S} P_{q n}^{S}}{\left(\frac{d_{p b}}{d_{p c}}\right)^{\alpha} I_{q n b}^{P}+\frac{B}{N} N_{o}}\right)\right],
$$

subject to:

$$
\begin{aligned}
& \sum_{q=1}^{Q} \sum_{n=1}^{N} z_{q n} \mathbb{E}\left[P_{q n}^{S}\right] \leq P_{\text {max }}, \\
& \sum_{q=1}^{Q} \sum_{n=1}^{N} z_{q n} I_{p n}^{S} \mathbb{E}\left[P_{q n}^{S}\right] \leq I_{p t}, \forall p \in P, \\
& P_{q n}^{S} \geq 0, \forall q \in Q, n \in N, \\
& z_{q n} \in\{0,1\}, \forall q \in Q, n \in N, \\
& \sum_{q=1}^{Q} z_{q n} \leq 1, \forall n \in N .
\end{aligned}
$$

In the above problem formulation, the constraint (27b) ensures that the total assigned power does not exceed the power limit $P_{\max }$ of the SBS. Similarly, the minimum assigned power should be a positive value which is enforced by constraint (27d). The interference caused by the CRN to a PU should remain below the threshold level and is guaranteed by (27c). Disjoint OFDMA subchannel assignment is ensured by (27e) and (27f) so that each subchannel is assigned to at most one user at a time for reliable communication.

\section{Subchannel And Power Allocation}

The optimization problem (27) is a mixed integer nonlinear programming (MINLP) problem, which is NP-hard and very challenging to solve. In order to arrive at a solution, we first relax the binary constraint $z_{q n} \in\{0,1\}$ to $z_{q n} \in[0,1]$. Then, a new variable $y_{q n}$ is introduced such that $y_{q n}=z_{q n} P_{q n}^{S}$. The transformed problem is given as follows:

$$
\begin{aligned}
\max _{y_{q n}, z_{q n}} & \frac{B}{N} \sum_{q=1}^{Q} \sum_{n=1}^{N} z_{q n} \mathbb{E}\left[\log _{2}\left(1+\frac{\psi_{q n} y_{q n}}{z_{q n}}\right)\right] \\
\text { subject to: } & \sum_{q=1}^{Q} \sum_{n=1}^{N} \mathbb{E}\left[y_{q n}\right] \leq P_{\max }, \\
& \sum_{q=1}^{Q} \sum_{n=1}^{N} I_{p n}^{S} \mathbb{E}\left[y_{q n}\right] \leq I_{p t}, \forall p \in P, \\
& \sum_{q=1}^{Q} z_{q n} \leq 1, \forall n \in N, \\
& y_{q n} \geq 0, \forall q \in Q, n \in N, \\
& z_{q n} \geq 0, \forall q \in Q, n \in N,
\end{aligned}
$$

where $\psi_{q n}=\frac{\left(\frac{d_{s b}}{d_{s c}}\right)^{\alpha} h_{q n b}^{S}}{\left(\frac{d_{p b}}{d_{p c}}\right)^{\alpha} I_{q n b}^{P}+\frac{B}{N} N_{o}}$. The objective function (28a) is concave with respect to $z_{q n}$ and $y_{q n}$, as it is the perspective function of a logarithmic function, which itself is concave. Further, all the constraints are affine. Hence, the transformed optimization problem (28) is a concave problem which can be solved using Karush-Kuhn-Tucker (KKT) conditions. The Lagrange function can be expressed as

$$
\begin{aligned}
\mathcal{L} & =\frac{B}{N} \sum_{q=1}^{Q} \sum_{n=1}^{N} z_{q n} \mathbb{E}\left[\log _{2}\left(1+\frac{\psi_{q n} y_{q n}}{z_{q n}}\right)\right] \\
& +\lambda\left(P_{\max }-\sum_{q=1}^{Q} \sum_{n=1}^{N} \mathbb{E}\left[y_{q n}\right]\right) \\
& +\sum_{p=1}^{P} \mu_{p}\left(I_{p t}-\sum_{q=1}^{Q} \sum_{n=1}^{N} I_{p n}^{S} \mathbb{E}\left[y_{q n}\right]\right) \\
& +\sum_{n=1}^{N} \eta_{n}\left(1-\sum_{q=1}^{Q} z_{q n}\right) .
\end{aligned}
$$

Differentiating this with respect to $y_{q n}$ results in,

$$
\frac{\partial \mathcal{L}}{\partial y_{q n}}=\frac{1}{\ln 2} \mathbb{E}\left[\frac{z_{q n} \psi_{q n}}{z_{q n}+\psi_{q n} y_{q n}}\right]-\lambda-\sum_{p=1}^{P} \mu_{p} I_{p n}^{S} .
$$

By setting the KKT condition $\frac{\partial \mathcal{L}}{\partial y_{q n}}=0$, we get

$$
y_{q n}=z_{q n}\left(\frac{1}{\ln 2\left(\lambda+\sum_{p=1}^{P} \mu_{p} I_{p n}^{S}\right)}-\frac{1}{\psi_{q n}}\right)^{+}
$$


where $[x]^{+}=\max \{0, x\}$. On the other hand, differentiating (29) with respect to $z_{q n}$, we have

$$
\begin{aligned}
\frac{\partial \mathcal{L}}{\partial z_{q n}} & =\mathbb{E}\left[\log _{2}\left(1+\frac{\psi_{q n} y_{q n}}{z_{q n}}\right)\right] \\
& -\frac{1}{\ln 2} \mathbb{E}\left[\frac{\psi_{q n} y_{q n}}{z_{q n}+\psi_{q n} y_{q n}}\right]-\eta_{n},
\end{aligned}
$$

which can be further expressed as follows by applying (31).

$$
\begin{aligned}
& \frac{\partial \mathcal{L}}{\partial z_{q n}} \\
& =\mathbb{E}\left[\log _{2}\left(1+\psi_{q n}\left(\frac{1}{\ln 2\left(\lambda+\sum_{p=1}^{P} \mu_{p} I_{p n}^{S}\right)}-\frac{1}{\psi_{q n}}\right)\right]\right. \\
& -\frac{1}{\ln 2} \mathbb{E}\left[\frac{\psi_{q n} z_{q n}\left(\frac{1}{\ln 2\left(\lambda+\sum_{p=1}^{P} \mu_{p} I_{p n}^{S}\right)}-\frac{1}{\psi_{q n}}\right)^{+}}{z_{q n}+\psi_{q n} z_{q n}\left(\frac{1}{\ln 2\left(\lambda+\sum_{p=1}^{P} \mu_{p} I_{p n}^{S}\right)}-\frac{1}{\psi_{q n}}\right)^{+}}\right]
\end{aligned}
$$$$
-\eta_{n}
$$

which equals to zero if $z_{q n}>0$, and is negative if $z_{q n}=0$. Furthermore, from the complementary slackness of KKT conditions, it follows that

$$
\begin{array}{r}
\lambda\left(P_{\max }-\sum_{q=1}^{Q} \sum_{n=1}^{N} \mathbb{E}\left[y_{q n}\right]\right)=0, \\
\mu_{p}\left(I_{p t}-\sum_{q=1}^{Q} \sum_{n=1}^{N} I_{p n}^{S} \mathbb{E}\left[y_{q n}\right]\right)=0, \forall p \in P, \\
\eta_{n}\left(1-\sum_{q=1}^{Q} z_{q n}\right)=0, \forall n \in N,
\end{array}
$$

with all Lagrangian multipliers $\lambda, \mu_{p}, \eta_{n} \geq 0$. From [7], it can be observed that under a practical assumption of distinct $\psi_{q n}$ values existing for all SUs, $\forall n \in N$, the transformed problem (28) can always achieve a binary optimal solution for the subcarrier allocation variable $z_{q n}$. Specifically, each subcarrier is solely assigned to the $\mathrm{SU}$ with the largest $\psi_{q n}$, i.e., $z_{q^{*} n}=1 \mid q^{*}=\arg \max \psi_{q n}$ and $z_{q n}=0 \mid q \neq q^{*}, \forall n$. The optimal values $z_{q n}^{*}$ and $y_{q n}^{*}$ for the transformed problem lead to $z_{q n}^{*}$ and $P_{q n}^{S *}=\frac{y_{q n}^{*}}{z_{q n}^{*}}$ which are optimal for the original problem.

After obtaining the optimal subcarrier allocation $z_{q n}^{*}$ using the aforementioned strategy, problem (28) becomes a locationassisted rate maximization problem with only power allocation required. As it is a concave problem, nearly any standard optimization method can reach a global optimum [12]. Specifically, since strong duality holds for a concave problem with Slater's constraint qualification satisfied, the optimal duality gap is zero and the Lagrangian dual problem can be solved. Hence, the subgradient method can be used to iteratively update the dual variables [12], as following:

$$
\begin{aligned}
& \lambda^{i+1}=\left(\lambda^{i}-\delta^{i}\left(P_{\max }-\sum_{q=1}^{Q} \sum_{n=1}^{N} \mathbb{E}\left[y_{q n}\right]\right)\right)^{+}, \\
& \mu_{p}^{i+1}=\left(\mu_{p}^{i}-\delta^{i}\left(I_{p t}-\sum_{q=1}^{Q} \sum_{n=1}^{N} I_{p n}^{S} \mathbb{E}\left[y_{q n}\right]\right)\right)^{+},
\end{aligned}
$$

where $\delta^{i}$ is a positive step size which is fixed independent of $i$ or is chosen following rules for the $i^{\text {th }}$ iteration [12]. Computation of both the subcarrier and power allocation is completed in linear time. Hence, the proposed resource allocation has overall linear time complexity.

\section{Simulation Results}

To simulate the proposed resource allocation scheme using MATLAB, we assume a CRN with a coverage radius of $500 \mathrm{~m}$. The CRN comprises of an SBS and 5 mobile SUs, unless otherwise indicated. The speed of each SU may vary from 0 to $100 \mathrm{Km} / \mathrm{h}$ and its direction may range from 0 to $2 \pi$ radians. We further assume that the SUs periodically send their sensing and position information to the SBS. Based upon this information, the SBS predicts the location and CSI of the SU for the next transmission slot. The maximum transmission power limit of the SBS, $P_{\max }$, is assumed to vary from $1 \mathrm{~W}$ to $1.5 \mathrm{~W}$. The number of PUs is assumed to be 4 . The number of OFDM subchannels, $N$, available to the CRN is 20 , and the bandwidth of each subchannel is $0.3125 \mathrm{MHz}$. The path loss exponent $\alpha$ is 4 . The interference threshold for a PU, $I_{p t}$, is assumed to be $5 \times 10^{-12} \mathrm{~W}$ [13]. Using an Intel Core i3-3110M 2.40-GHz CPU with 4 GB RAM, the average time taken by the proposed resource allocation strategy varies from 1.1 to $1.5 \mathrm{~s}$.

The effect of change in speeds of SUs on their total capacity is shown in Fig. 2. The decrease in data rate with increase in speed can mainly be attributed to multipath fading and Doppler spread. In particular, Doppler shift increases with velocity resulting in impairment of the wireless channel. As concluded in [14], increased time variations of the channel adversely affect the orthogonality of different OFDM subcarriers giving rise to inter carrier interference (ICI). This results in performance degradation of the system.

In Fig. 3, we plot the total capacity of SUs versus the total transmission power limit of the SBS for three different average velocities of SUs, i.e. 10,50 and $100 \mathrm{Km} / \mathrm{h}$. As can be observed from Fig. 3, the total capacity of the CRN increases as the maximum transmission power limit of the SBS increases. However, it decreases with an increase in the average speed of SUs as is also evident from Fig. 2.

We compare our predictive allocation with the static allocation, which does not consider mobility, for two values of 


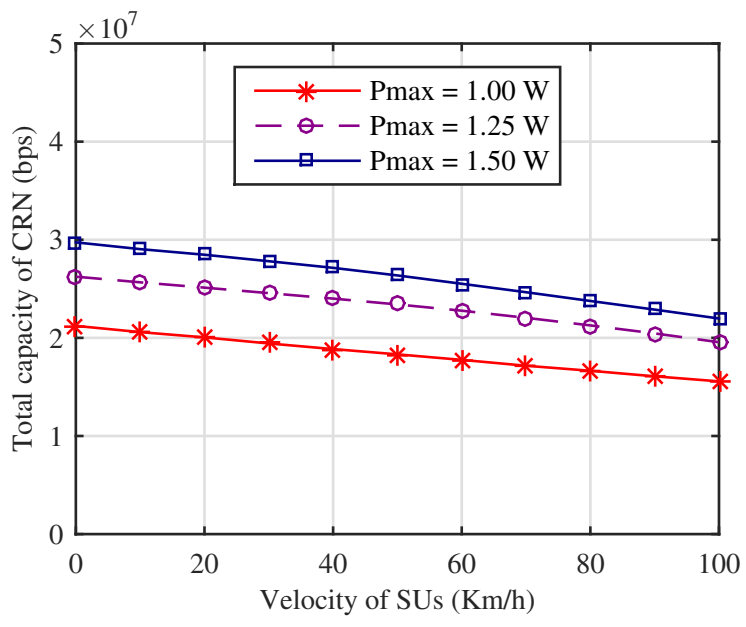

Fig. 2. Total capacity of CRN versus the average velocity of SUs.

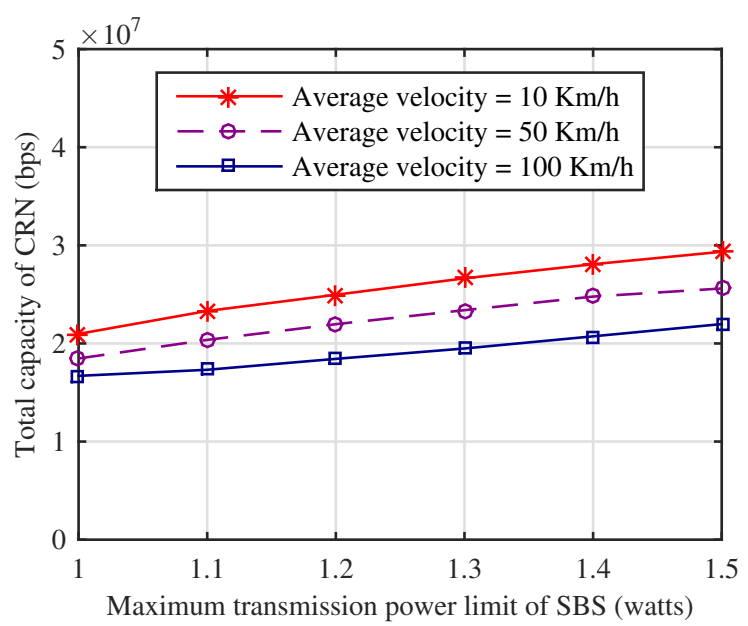

Fig. 3. Total capacity of CRN versus the maximum transmission power.

$P_{\max }$. Performance of the static allocation scheme degrades more rapidly with increase in average velocity of SUs as seen in Fig. 4. The predictive allocation is able to predict the CSI for the next transmission slot and hence a better allocation is possible. In the case of static allocation, the allocation is based upon the previous CSI results available at the time of allocation. Meanwhile, the SU may have traveled to a different location where the CSI is different from the one upon which the allocation was based. This results in degradation of the performance of the static allocation with increase in velocity.

\section{CONCLUSION}

We investigated location-assisted resource allocation problem for the downlink transmission in a mobile CRN with multiple OFDMA subchannels. An MINLP optimization problem was formulated based upon the location cognitive ability of the CRN and an optimal solution for subcarrier and power allocation was obtained. Simulation results confirm the effectiveness of the proposed solution based upon CSI prediction compared to static allocation which does not consider mobility.

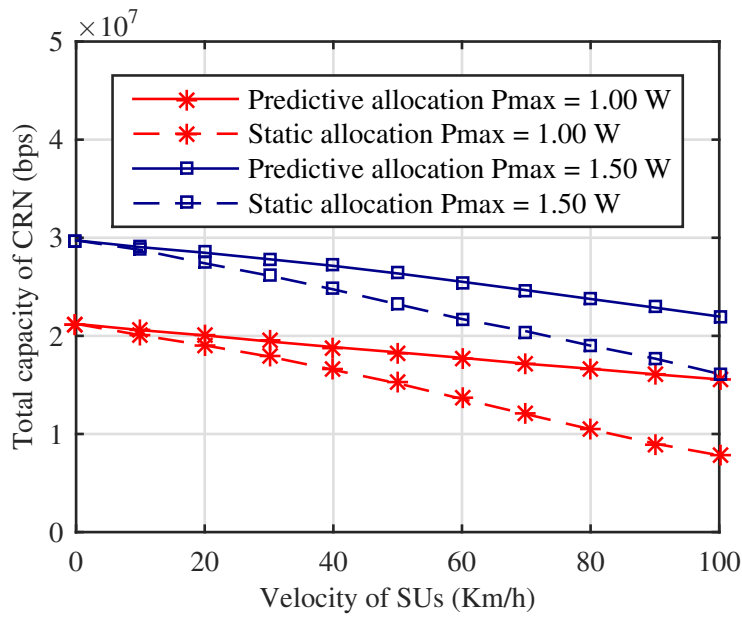

Fig. 4. Comparison of predictive and static allocation schemes.

\section{REFERENCES}

[1] S. Rajendran, R. Calvo-Palomino, M. Fuchs, B. V. den Bergh, H. Cordobes, D. Giustiniano, S. Pollin, and V. Lenders, "Electrosense: Open and big spectrum data," IEEE Communications Magazine, vol. 56, no. 1, pp. 210-217, Jan 2018.

[2] Q. Ni and C. C. Zarakovitis, "Nash bargaining game theoretic scheduling for joint channel and power allocation in cognitive radio systems," IEEE Journal on Selected Areas in Communications, vol. 30, no. 1, pp. 70-81, January 2012.

[3] Z. Zhou, M. Dong, K. Ota, G. Wang, and L. T. Yang, "Energy-efficient resource allocation for D2D communications underlaying cloud-RANbased LTE-A networks," IEEE Internet of Things Journal, vol. 3, no. 3, pp. 428-438, June 2016.

[4] Z. Zhou, K. Ota, M. Dong, and C. Xu, "Energy-efficient matching for resource allocation in D2D enabled cellular networks," IEEE Transactions on Vehicular Technology, vol. 66, no. 6, pp. 5256-5268, June 2017.

[5] J. V. Hecke, P. D. Fiorentino, V. Lottici, F. Giannetti, L. Vandendorpe, and M. Moeneclaey, "Distributed dynamic resource allocation for cooperative cognitive radio networks with multi-antenna relay selection," IEEE Transactions on Wireless Communications, vol. 16, no. 2, pp. 1236-1249, Feb 2017.

[6] H. Hu, H. Zhang, and N. Li, "Location-information-assisted joint spectrum sensing and power allocation for cognitive radio networks with primary-user outage constraint," IEEE Transactions on Vehicular Technology, vol. 65, no. 2, pp. 658-672, Feb 2016.

[7] X. Wang, S. Ekin, and E. Serpedin, "Joint spectrum sensing and resource allocation in multi-band-multi-user cognitive radio networks," IEEE Transactions on Communications, vol. 66, no. 8, pp. 3281-3293, 2018.

[8] T. Xue, X. Dong, and Y. Shi, "Resource-allocation strategy for multiuser cognitive radio systems: Location-aware spectrum access," IEEE Transactions on Vehicular Technology, vol. 66, no. 1, pp. 884-889, 2017.

[9] "Facilitating opportunities for flexible, efficient, and reliable spectrum use employing cognitive radio technologies," Federal Communications Commission, Tech. Rep. FCC-05-57, March 2005.

[10] N. Saeed and H. Nam, "Energy efficient localization algorithm with improved accuracy in cognitive radio networks," IEEE Communications Letters, vol. 21, no. 9, pp. 2017-2020, Sept 2017.

[11] T. Weiss, J. Hillenbrand, A. Krohn, and F. K. Jondral, "Mutual interference in OFDM-based spectrum pooling systems," in 2004 IEEE 59th Vehicular Technology Conference., vol. 4, May 2004, pp. 1873-1877.

[12] S. Boyd and L. Vandenberghe, Convex Optimization. Cambridge Univ. Press, 2004

[13] M. Liu, T. Song, J. Hu, H. Sari, and G. Gui, "Anti-shadowing resource allocation for general mobile cognitive radio networks," IEEE Access, vol. 6, pp. 5618-5632, 2018.

[14] T. Wang, J. G. Proakis, E. Masry, and J. R. Zeidler, "Performance degradation of OFDM systems due to Doppler spreading," IEEE Transactions on Wireless Communications, vol. 5, no. 6, pp. 1422-1432, June 2006. 